\title{
Algorithmic Foundations of Realizing Multi-Contact Locomotion on the Humanoid Robot DURUS
}

\author{
Jacob P. Reher, Ayonga Hereid, Shishir Kolathaya, Christian M. Hubicki and \\ Aaron D. Ames \\ Georgia Institute of Technology, Atlanta, GA, 30332
}

\begin{abstract}
This paper presents the meta-algorithmic approach used to realize multi-contact walking on the humanoid robot, DURUS. This systematic methodology begins by decomposing human walking into a sequence of distinct events (e.g. heel-strike, toe-strike, and toe push-off). These events are converted into an alternating sequence of domains and guards, resulting in a hybrid system model of the locomotion. Through the use of a direct collocation based optimization framework, a walking gait is generated for the hybrid system model emulating human-like multi-contact walking behaviors - additional constraints are iteratively added and shaped from experimental evaluation to reflect the machine's practical limitations. The synthesized gait is analyzed directly on hardware wherein feedback regulators are introduced which stabilize the walking gait, e.g., modulating foot placement. The end result is an energyoptimized walking gait that is physically implementable on hardware. The novelty of this work lies in the creation of a systematic approach for developing dynamic walking gaits on 3D humanoid robots: from formulating the hybrid system model to gait optimization to experimental validation refined to produce multi-contact $3 \mathrm{D}$ walking in experiment.
\end{abstract}

\section{Introduction}

Biological bipeds, such as humans, demonstrate walking patterns which are efficient, agile, fast, and robust to a degree not yet attainable by robotic systems. While humans and other biological bipeds can perform these motions with relative ease, translation of these capabilities to $3 \mathrm{D}$ humanoid systems is fraught with complexities in the form of nonlinearities, modeling errors, and high degrees of freedom which must be coordinated. With the goal bridging this gap in natural and efficient locomotion on robots, it is advantageous develop algorithmic approaches capable of exploiting the natural dynamics of the robot. While some researchers argue robotics is currently limited by physical hardware capabilities, a lack of fundamental knowledge in the area has yet to be bridged as well. Robotic walking presents a wide range of mathematical and algorithmic challenges that provides an fertile proving ground for addressing these gaps.

Many of the approaches currently employed revolve around the use of reduced order inverted pendulum models. Perhaps the most prevalent approach 


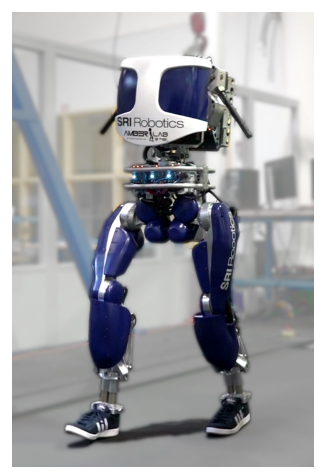

(a) DURUS

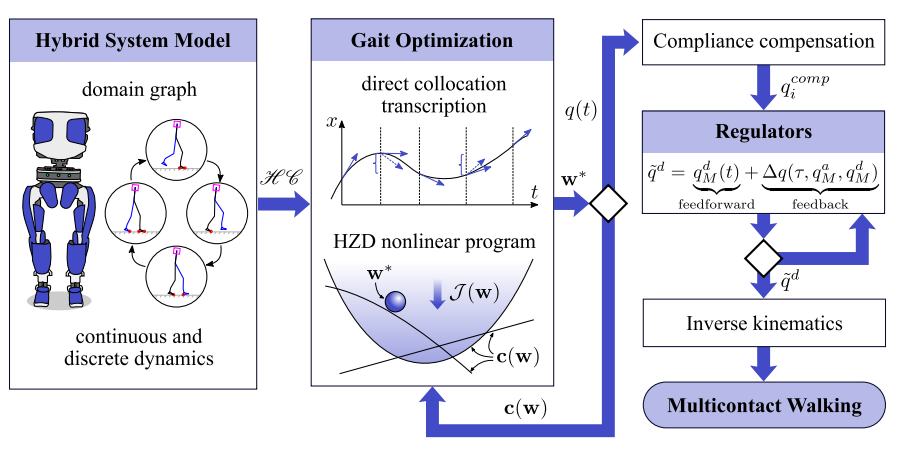

(b) "Meta-algorithm" for gait development

Fig. 1: (a) The humanoid robot, DURUS, walking heel-to-toe. (b) The "metaalgorithm" followed to achieve walking control, with decision points (diamonds) representing iteration based on the results of robot experiments.

uses a framework known as the Zero Moment Point (ZMP) criterion [19]. The resulting walking motions are typically flat footed and quasi-static. Human walking patterns, on the other hand, consist of multiple phases (or domains) with changes in contact conditions, impacts, and underactuation [18]. In an attempt to generate more human-like walking patterns, multi-contact methods have been implemented which allowed for longer walking strides and increased energy efficiency through heel and toe contact conditions $[7,15]$. One difficulty with this approach is that its inherent assumptions prevent it from utilizing the natural forward momentum of the robot in a manner similar to humans.

A method which has been used to generate dynamic walking motions with stability guarantees through underactuated domains is termed Hybrid Zero Dynamics (HZD) $[3,9,21]$. The stability of these methods on bipeds has been validated experimentally [17], and it has also been shown that HZD methods can be extended to 3D robots [16]. However, to the authors knowledge, HZD methods have not been utilized to obtain multi-contact walking behaviors on a 3D humanoid robot with both heel-toe contact motions and underactuation.

The goal of this paper is to provide a foundation upon which HZD based multi-contact walking behaviors can be formally generated and then experimentally realized on humanoid robots. With this goal in mind, we begin with a discussion of human walking patterns and their relation to the domain structure hybrid model of humanoid walking in Sec. 2. The optimization method, including the cost function and constraints necessary to arrive at an experimentally realizable gait, are presented in Sec. 3. The experimental methods and results along with the discrete feedback compensation algorithms used for experimental stabilization are presented in Sec. 4, in which a mean cost of transport over 200 steps is shown as 1.02, the lowest electrical cost of transport yet reported on a 3D humanoid robot. Finally, an analysis of the overall methodology performance for multi-contact gait generation is presented in Sec. 5 . 


\section{Robot Walking Model}

In this section, we will discuss human walking and the corresponding partitioning of the walking behavior into the domains heel strike, toe strike, toe lift, and heel lift. This four-domain structure is incorporated for the robotic model as a hybrid system with an alternating sequence of continuous and discrete events.

\subsection{Human Walking Domains}

In studies of human locomotion, multi-contact behaviors have been found to be essential to reducing joint torques and increasing walking speeds [11]. In this work, a walking gait for a 3D humanoid robot is designed with a hybrid domain breakdown matching that of the temporal domain pattern observed in natural human walking motions [4]. From Fig. 2 it can be observed that human walking has four distinct phases: heel strike $(h s)$ when the swinging foot strikes the ground, toe strike $(t s)$ when the toe of the foot goes down and the legs switch, toe lift $(t l)$ when the other foot takes off the ground and becomes the swinging foot and finally heel lift $(h l)$ when the stance heel goes up with the stance toe being the only contact point with ground. The behavior goes from fully actuated (hs) to overactuated (ts) to fully actuated (tl) to underactuated (hl) phases of motion in a sequence in a repeated manner where a domain is considered underactuated or overactuated if the humanoid has less or more actuators and contact constraints than degrees of freedom in the system.

What determines the phase in which the robot is currently operating is the set of contact points $\mathcal{C}=\{s w h, s w t, s t h, s t t\}$ (swing heel, swing toe, stance heel, stance toe). The switch of each leg from stance to swing and vice versa occurs after toe strike (see Fig. 2). Recent work by the authors [22] detailed the implementation of multi-contact locomotion on two $2 \mathrm{D}$ robots. In this work,

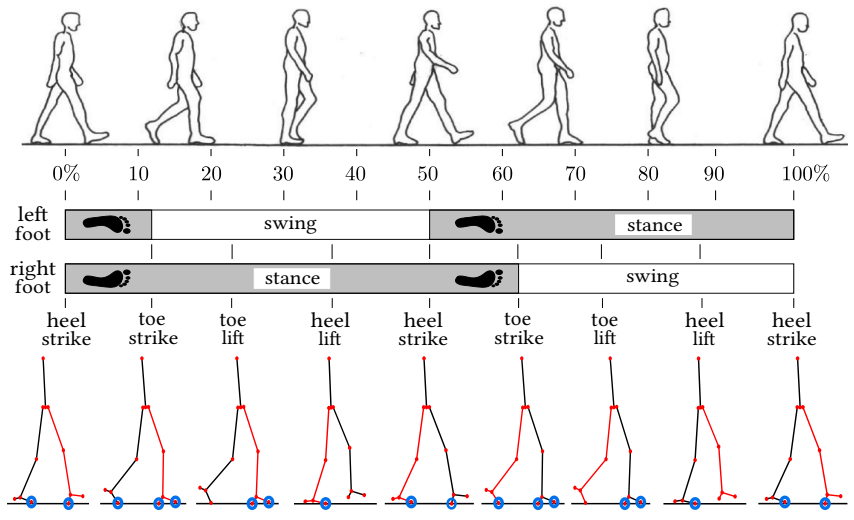

Fig. 2: Multi-contact locomotion diagram of a typical human gait cycle [2] (top) and multi-contact domain breakdown of two steps of one subject based on the changes of heel and toe contact condition (bottom). Blue circles represent one specific point in contact with the walking surface. 
three domains were used to represent locomotion, with the removal of the domain corresponding to the toe liftoff before swing $(t s)$. While this domain is relatively short in comparison to the overall gait cycle, the inclusion of this phase allows for walking which is more closely aligned with human locmotion and permits longer steps. This four domain representation of human walking is next framed in the context of constructing a hybrid system model.

\subsection{Multi-Domain Hybrid System}

Bipedal robots display both continuous and discrete behaviors, lending themselves naturally to hybrid systems models. Continuous evolution of the biped dynamics occurs when there is a fixed number of contact points with the environment, in which we say the robot is on a vertex. There is a discrete change in the biped dynamics when the number of contact points with the environment changes, on which we say the robot has reached an edge. The study of human walking data shows how a periodic human-like walking gait can be described by a directed cycle based on contact conditions. The directed cycle is given by a graph $\Gamma=(V, E)$ consisting of four vertices and edges:

$$
V=\{t s, t l, h l, h s\}, E=\{t s \rightarrow t l, t l \rightarrow h l, h l \rightarrow h s, h s \rightarrow t s\},
$$

where each vertex represents a continuous domain and each edge corresponds to a transition between these domains, as shown in Fig. 3. For example, ts denotes the toe strike and the corresponding vertex denotes all possible states where the toe is in contact with the ground. Motivated by this breakdown, we adapt this human like multi-domain formulation with four vertices and edges. Interested readers are referred to $[4,22]$ for a full definition of the multi-domain hybrid system. For the $n$-DOF robot of configuration $q \in \mathcal{Q} \subset \mathbb{R}^{n}$, tangent bundle with (local) coordinates $(q, \dot{q}) \in T \mathcal{Q} \subset \mathbb{R}^{2 n}$, the multi-domain hybrid control system is defined to be a tuple:

$$
\mathscr{H} \mathscr{C}=(\Gamma, \mathcal{D}, \mathcal{U}, S, \Delta, F G) .
$$

- $\Gamma$ is the directed cycle specified by (1).

$-\mathcal{D}=\left\{\mathcal{D}_{v}\right\}_{v \in V}$ is the set of domains of admissibility. Each domain $\mathcal{D}_{v} \subseteq X \times U$ with $X \subseteq \mathbb{R}^{2 n}$ can be interpreted as the set of possible states the robot can assume given the constraints on the feet for the corresponding domain. For example,

$$
\mathcal{D}_{t s}=\left\{(q, \dot{q}) \in \mathbb{R}^{2 n} \mid\left[h_{s t t}, h_{s t h}, h_{s w t}\right]^{T}=0, h_{s w h} \geq 0\right\},
$$

where $h_{s w h}, h_{s w t}, h_{s t h}$, and $h_{s t t}$ are the vertical positions of the foot contact points. An alternative definition of the domain can also be obtained by using the holonomic constraints $h_{v}: \mathcal{Q} \rightarrow \mathbb{R}^{l}$ wherein the position and orientation of the contact points $\mathcal{C}$ are fixed.

- $S=\left\{S_{t s \rightarrow t l}, S_{t l \rightarrow h l}, S_{h l \rightarrow h s}, S_{h s \rightarrow t s}\right\}$ is the set of guards which form the transition point from one domain to another. For example,

$$
S_{h l \rightarrow h s}=\left\{(q, \dot{q}) \in \mathbb{R}^{2 n} \mid\left[h_{s t t}, h_{s w h}\right]^{T}=0, \dot{h}_{s w h} \leq 0\right\} .
$$


$-\mathcal{U}$ is the set of admissible control inputs.

$-\Delta=\left\{\Delta_{t s \rightarrow t l}, \Delta_{t l \rightarrow h l}, \Delta_{h l \rightarrow h s}, \Delta_{h s \rightarrow t s}\right\}$ is the set of reset maps from one domain to the next domain. In the presence of an impact, the reset map emits the post impact state of the robot. Each reset map $\Delta_{e}: S_{e} \rightarrow \mathcal{D}_{v_{\text {target }}}$, with $e=\left\{v_{\text {source }} \rightarrow v_{\text {target }}\right\} \in E$ is computed by assuming that the impacts are plastic and instantaneous [10].

- $F G$ provides the set of vector fields given by the equation: $\dot{x}=f_{v}(x)+g_{v}(x) u$, where $x=(q, \dot{q}), u \in \mathcal{U} . f_{v}, g_{v}$ are defined in each domain by the EulerLagrangian dynamics. More details on the dynamics are given below.

Given the state $(q, \dot{q}) \in T \mathcal{Q}$, the dynamics of the system with foot contact constraints for each domain are given by:

$$
\begin{aligned}
D(q) \ddot{q}+H(q, \dot{q})-B u-J_{v}^{T}(q) \lambda_{v} & =0, \\
J_{v}(q) \ddot{q}+\dot{J}_{v}(q, \dot{q}) \dot{q} & =0
\end{aligned}
$$

where $D, H$ have the usual meaning from the Euler-Lagrangian dynamics, $B$ is the mapping of torques to the joints, $\lambda_{v}$ is the set of ground reaction forces and $J_{v}$ is the Jacobian for the contact points where $\lambda_{v}$ is applied.

\section{Direct Collocation Based HZD Optimization}

Using the hybrid system model, a gait optimization framework is now introduced which is used to generate walking gaits for the robot with a set of parameters to yield a hybrid invariant periodic orbit. This guarantees that at least for simulation, the bipedal walking is stable. This comprises the next element in the "meta-algorithm", in which these walking gaits are obtained, recorded, and tested on hardware. In the authors' previous work, a direct collocation formulation of HZD gait optimization has been successfully applied to DURUS to generate flat-footed walking gaits [8]. In this paper, we extend this methodology

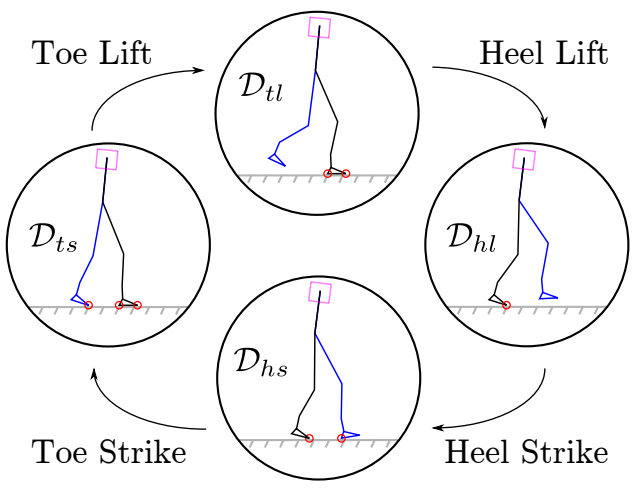

Fig. 3: The directed cycle of four domains for $3 \mathrm{D}$ multi-contact walking. The red circles represent foot contact points.

\begin{tabular}{lll}
\hline Domain & Human & DURUS \\
\hline $\mathcal{D}_{t s}$ & $6 \%$ & $4.6 \%$ \\
$\mathcal{D}_{t l}$ & $59 \%$ & $52.4 \%$ \\
$\mathcal{D}_{h l}$ & $18 \%$ & $29.6 \%$ \\
$\mathcal{D}_{h s}$ & $17 \%$ & $13.4 \%$ \\
\hline
\end{tabular}

Table 1: A comparison of domain durations which has been shown to be consistent in human locomotion versus the walking gait implemented on DURUS through optimization. 
to the multi-contact case. Similar methods have been proposed utilizing constrained dynamical systems with open loop controllers to achieve multi-contact walking gaits in simulation [13].

Feedback Linearization. The feedback linearizing controller introduced in this section allows for the formulation of a set of stability criteria used throughout the optimization. Specifically, to lend itself to formal analysis, we must set up a problem in which we can theoretically drive the actual robot configuration to the desired outputs of the system. We have the set of actual outputs of the robot as $y_{a}: T \mathcal{Q} \rightarrow \mathbb{R}^{2 n}$, and the desired outputs as $y_{d}: \mathbb{R}^{+} \rightarrow \mathbb{R}^{m} \cdot y_{d}$ is usually modulated by a phase (or time) variable $\tau: \mathcal{Q} \rightarrow \mathbb{R}^{+}$(or $\tau: \mathbb{R}^{+} \rightarrow \mathbb{R}^{+}$ for time based). By adapting a feedback linearizing controller, we can drive the relative degree one outputs $y_{1, v}(q, \dot{q})=y_{a, v}^{1}(q, \dot{q})-y_{d, v}^{1}\left(\tau, \alpha_{v}\right)$ and relative two outputs $y_{2, v}(q)=y_{a, v}^{2}(q)-y_{d, v}^{2}\left(\tau, \alpha_{v}\right)$ to zero, with $v$ denoting the domain, $\alpha$ denoting the parameters of the desired trajectory. These outputs are generally called virtual constraints [20]. Note that any effective tracking controller will theoretically suffice (the experimental implementation uses PD control [9]). The feedback linearizing controller [4] that drives $y_{1, v} \rightarrow 0, y_{2, v} \rightarrow 0$ is given by:

$$
u_{v}=\left[\begin{array}{c}
L_{g} y_{1, v} \\
L_{g} L_{f} y_{2, v}
\end{array}\right]^{-1}\left(-\left[\begin{array}{c}
L_{f} y_{1, v} \\
L_{f}^{2} y_{2, v}
\end{array}\right]+\mu_{v}\right)
$$

where $L_{g}, L_{f}$ denote the Lie derivatives and $\mu_{v}$ denotes the auxiliary input applied after the feedback linearization. Applying $u_{v}$ in (6) yields linear dynamics:

$$
\dot{\eta}_{v}(q, \dot{q})=\mu_{v}
$$

where $\dot{\eta}_{v}:=\left[\dot{y}_{1, v}, \ddot{y}_{2, v}\right]^{T}$, so that $\mu_{v}$ can be carefully chosen to stabilize the output dynamics.

Partial Hybrid Zero Dynamics. When the control objective is met such that $y_{1, v}, y_{2, v}=0$ for all time then the system is said to be operating on the partial zero dynamics surface [3] (full zero dynamics for purely relative degree two outputs):

$$
\mathbb{Z}_{v}=\left\{(q, \dot{q}) \in \mathcal{D}_{v} \mid y_{2, v}=0, L_{f} y_{2, v}=0\right\}
$$

for the domain $\mathcal{D}_{v}$. The controller $u_{v}$, being domain specific, guarantees partial zero dynamics only in the continuous dynamics. Therefore, for a multi-domain hybrid system, partial hybrid zero dynamics can be guaranteed if and only if the discrete maps $\Delta_{e}$ are invariant of the partial zero dynamics in each domain. As a result, the parameters $\alpha_{v}$ of the outputs must be chosen in a way which renders the surface invariant through impacts. can be mathematically formulated as:

$$
\Delta_{e}\left(\mathbb{Z}_{v_{\text {source }}} \cap S_{e}\right) \subset \mathbb{Z}_{v_{\text {target }}}, \quad e=\left\{v_{\text {source }} \rightarrow v_{\text {target }}\right\} \in E .
$$

The best way to ensure hybrid invariance under a discrete transition is by a careful selection of the desired trajectories (desired gait) via the parameterization: 
$y_{d, v}\left(\tau, \alpha_{v}\right)$. Hence if the desired trajectories are a function of Bezier polynomials, the parameters $\alpha_{v}$ are the coefficients. These coefficients are chosen by using a direct collocation based walking gait optimization problem explained in the following section.

Collocation Algorithm. Here, we simply introduce the main idea of the direct collocation optimization [8]. In particular, the solution of each domain, $\mathcal{D}_{v}$, is discretized based on the time discretization $0=t_{0}<t_{1}<t_{2}<\cdots<t_{N_{v}}=T_{I, v}$, assuming $T_{I, v}>0$ is the time at which the system reaches the guard associated with a given domain. Let $x^{i}$ and $\dot{x}^{i}$ be the approximated states and first order derivatives at node $i$, the defect constraints are defined at each odd node as:

$$
\begin{aligned}
& \zeta^{i}:=\dot{x}^{i}-3\left(x^{i+1}-x^{i-1}\right) / 2 \Delta t_{v}^{i}+\left(\dot{x}^{i-1}+\dot{x}^{i+1}\right) / 4=0, \\
& \delta^{i}:=x^{i}-\left(x^{i+1}+x^{i-1}\right) / 2-\Delta t_{v}^{i}\left(\dot{x}^{i-1}-\dot{x}^{i+1}\right) / 8=0,
\end{aligned}
$$

where $\Delta t_{v}^{i}=t_{i+1}-t_{i-1}$ is the time interval. Moreover, the first order derivatives must satisfy the system dynamics, i.e., the restricted partial zero dynamics in the context of HZD.

Constrained Hybrid Dynamics. Recall that reduced dimensional restricted dynamics, i.e., the zero dynamics, is determined via the full order dynamics (5) subject to the holonomic constraints and virtual constraints being zero. Thus, the restricted dynamics can be described as the differential algebraic equations:

$$
F_{v}\left(q, \dot{q}, \ddot{q}, u, \lambda_{v}, \alpha_{v}\right):=\left[\begin{array}{l}
D(q) \ddot{q}+H(q, \dot{q})-B u-J_{v}^{T}(q) \lambda_{v} \\
J_{v}(q) \ddot{q}+\dot{J}_{v}(q, \dot{q}) \dot{q} \\
\dot{\eta}_{v}\left(q, \dot{q}, \ddot{q}, \alpha_{v}\right)-\mu_{v}
\end{array}\right]=0
$$

subject to the initial value conditions:

$$
\begin{aligned}
h_{v}\left(q^{+}\right) & =\bar{h}_{v}, \quad J_{v}\left(q^{+}\right) \dot{q}^{+}=0, \\
y_{2, v}\left(q^{+}\right) & =0, \quad \dot{y}_{2, v}\left(q^{+}, \dot{q}^{+}\right)=0,
\end{aligned}
$$

where $\bar{h}_{v}$ is a vector of constants, and $\left(q^{+}, \dot{q}^{+}\right)$are the initial state values. This system can be considered as an implicit form that is equivalent to the zero dynamics equation by its definition.

Moreover, the trajectories of the system states of two neighboring domains are connected via the discrete dynamics captured in the reset maps. Specifically, suppose that $\left(q^{+}, \dot{q}^{+}\right)$are the post-impact states of a particular domain and $\left(q^{-}, \dot{q}^{-}\right)$are the pre-impact states of the previous domain, then they must satisfy

$$
\left(q^{+}, \dot{q}^{+}\right)-\Delta_{e}\left(q^{-}, \dot{q}^{-}\right)=0
$$

where $e \in E$ corresponds to the transition between these two domains. This constraint together with the initial value constraint in (14) guarantee that the hybrid invariant conditions are satisfied, therefore, the solution to the optimization lies on the partial hybrid zero dynamics manifold given in (8). 
General Formulation. Let $\mathbf{w}$ be a vector containing all optimization variables and $\mathbf{c}(\mathbf{w})$ be a vector of constraint functions given in (10) - (15), we then state the optimization problem as,

$$
\begin{aligned}
\mathbf{w}^{*}=\underset{\mathbf{w}}{\operatorname{argmin}} & \mathcal{J}(\mathbf{w}) \\
\text { s.t } & \mathbf{c}^{\min } \leq \mathbf{c}(\mathbf{w}) \leq \mathbf{c}^{\max }, \\
& \mathbf{w}^{\min } \leq \mathbf{w} \leq \mathbf{w}^{\max }
\end{aligned}
$$

Cost Function. Despite the goal of human-like walking, we do not impose any human specific constraints in the optimization. Instead, we define the cost function of our gait optimization as minimizing the total mechanical cost of transport of the gait:

$$
\mathcal{J}:=\frac{1}{m g l_{\text {step }}} \sum_{v \in V} \int_{t_{v}^{+}}^{t_{v}^{-}} P(\dot{q}(t), u(t)) d t
$$

where $t_{v}^{+}$and $t_{v}^{-}$is the initial and final time of a domain $v, m g$ is the weight of the robot and $l_{\text {step }}$ is the step length of one gait cycle, respectively, and $P(\dot{q}(t), u(t))$ is the 2-norm sum of the mechanical power, given as

$$
P(\dot{q}(t), u(t)):=\sum_{j=1}^{m}\left\|u_{j}(t) \cdot \dot{q}_{j}(t)\right\|
$$

where $u_{j}(t)$ and $\dot{q}_{j}(t)$ is the computed torque and joint velocity of each actuated joint $j$. In the context of the direct collocation optimization, the numerical integration in (17) is computed with the discrete state and control variables using quadrature rules [8].

Physical Constraints. In addition to the constraints defined in (10) - (15), other physical constraints can be easily added into $\mathbf{c}$ to ensure the resulting gaits are feasible on hardware. For example, torque bounds, joint velocity limits and angle limits, etc., can be imposed directly as the boundary value of corresponding optimization variables in $\mathbf{w}^{\min }$ or $\mathbf{w}^{\max }$. Hence, the method lends itself naturally to the addition of physical constraints based on actual hardware considerations for the physical hardware. Using this approach, the following constraints are added to the gait optimization and are configured specifically to provide favorable conditions for experimental walking.

- Torso Movement. The optimization tends to find energetically minimal walking gaits in which the torso inertia is used similar to arm-swing to counter moments generated by the swinging legs. When implemented experimentally, gaits with particularly large torso swing tend to worsen unwanted contact conditions, such as loss of foot contacts or early striking. This can be prevented by constraining the torso movement in the gait design. Let $\phi_{\text {tor }}(q): \mathcal{Q} \rightarrow \mathbb{R}^{3}$ be the three dimensional orientations of the upper torso link, we restrict them within a small range $\left[\phi_{t o r}^{\min }, \phi_{\text {tor }}^{\max }\right]$, i.e.,

$$
\phi_{\text {tor }}^{\min } \leq \phi_{\text {tor }}(q) \leq \phi_{\text {tor }}^{\max }
$$


- Impact Velocity. If the swing foot impacts the ground too hard, it can destabilize the robot. Therefore, we constrain the impact velocities of the heel to be within a reasonable range. Let $v_{x}^{\max }, v_{y}^{\max }, v_{z}^{\max }>0$ be the maximum allowable impact velocities in $x, y$, and $z$ direction respectively, then the swing heel velocities $\dot{h}_{\text {swh }}\left(q^{-}, \dot{q}^{-}\right)$should satisfy

$$
\left|\dot{h}_{s w h}^{x}\left(q^{-}, \dot{q}^{-}\right)\right| \leq v_{x}^{\max },\left|\dot{h}_{s w h}^{y}\left(q^{-}, \dot{q}^{-}\right)\right| \leq v_{y}^{\max },\left|\dot{h}_{s w h}^{z}\left(q^{-}, \dot{q}^{-}\right)\right| \leq v_{z}^{\max }
$$

where $\left(q^{-}, \dot{q}^{-}\right) \in \mathcal{D}_{h l} \cap S_{h l \rightarrow h s}$.

- Swing Leg Roll. Due to the existence of unmeasured compliance in the mechanical system, the swing leg can strike the stance leg if they are not separated enough. The separation of legs can be expressed as the difference between stance and swing hip roll angles. Assuming the right leg is the stance leg, then the following constraint should be enforced:

$$
\phi^{\min } \leq \phi_{r h}-\phi_{l h} \leq \phi^{\max }
$$

where $\phi_{r h}$ and $\phi_{l h}$ are the right and left hip roll angles, and $\phi^{\max }>\phi^{\min } \geq 0$ are the maximum and minimum allowable leg separation angles.

- Ground Reaction Wrench Constraints. In Sec. 2, we model the ground-foot contact as holonomic constraints. However, these constraints are unilateral in essence. Thus the ground reaction wrenches resulting from the contact conditions cannot be infinitely large. The limitations of ground reaction wrenches are often described as the Zero Moment Point (ZMP) constraints, which are discussed thoroughly in [6]. In particular, we enforce the ZMP constraints only during the single support domain $\mathcal{D}_{t l}$ when the stance foot is flat on the ground. In addition, we also constrain the yaw reaction moment of the stance foot, $\lambda_{s f}^{m z}$, to be reasonably small:

$$
\left\|\lambda_{s f}^{m z}\right\| \leq \lambda^{\max }
$$

where $\lambda^{\max }$ is the maximum acceptable yaw reaction moment.

\section{Experimental Validation}

The main goal of this work is to allow for the formal generation of HZD based multi-contact walking gaits which can then be rapidly prototyped on hardware to ensure suitability for the application of more advanced control methods. This section details the implementation method used to validate the suitability of gaits generated via the methods proposed in Sec. 3 on hardware. Desired motor trajectories are interpolated through the time based trajectory generated using the formal methods detailed in Sec. 3 and played back as a feedforward element. The joint configuration of the robot is then adjusted as:

$$
\tilde{q}^{d}=\underbrace{q_{M}^{d}\left(t, \alpha_{v}\right)}_{\text {feedforward }}+\underbrace{\Delta q\left(\tau, \alpha_{v}, x_{M}^{a}\right)}_{\text {feedback }},
$$

wherein three compensators are used as feedback. 


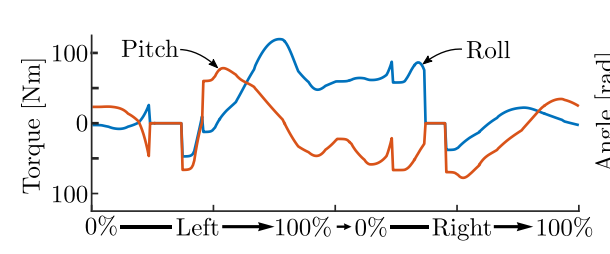

(a)

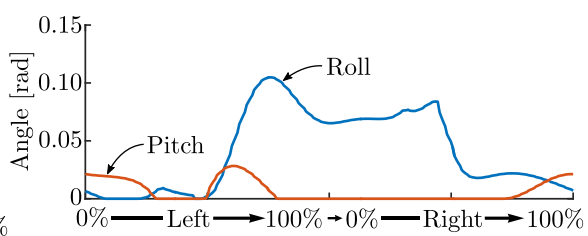

(b)

Fig. 4: (a) The anticipated torque computed on the robot using the time-based trajectories over two steps. (b) Angle compensation applied directly to the hip roll and pitch joints over the corresponding steps.

\subsection{Experimental Methods}

In order to ensure the robot is able to compensate for small modeling uncertainties during gait evaluation, we introduce three compensators which comprise the experimental implementation aspect of the "meta-algorithmic" approach. These feedback actions manifest as small modifications to the joint angle commands correcting for unmeasured compliance, lateral lean, and heel strike orientation.

Compliance Compensation. A prevalent problem with humanoid hardware is unmeasured compliance in the system. Throughout the walking behaviors presented in this work, DURUS exhibited unmeasured compliance in its hips with deflections reaching over 0.1 radians. This has also been cited as an issue on ATLAS [12], in which a linear compliance assumption is introduced to augment the measured angles fed to a fullbody estimator. A similar approach is used here with the primary difference that the compensator directly adjusts the desired joint configuration via a position command:

$$
q_{i}^{\text {comp }}=q_{M, i}^{d}+u_{i} / K_{i},
$$

where $i \in \mathcal{Q}$ are the corresponding joints, $q_{i}^{\operatorname{comp}}$ is the preprocessed joint angle to be passed to the controller, $q_{M, i}^{d}$ is the feedforward joint angle, $u_{i}$ is the feedback linearizing torque computed at each time step, and $K_{i}$ is the stiffness coefficient which has been measured for each joint. The compliance parameters, measured with a force gauge and caliper in units of $\mathrm{Nm} / \mathrm{rad}$, for the worst case joints on DURUS were found to be $K_{l h p}=1284, K_{l h r}=900, K_{r h p}=1124$, and $K_{r h r}=815$. The anticipated torque at the joint is computed online and the values $q_{i}^{\text {comp }}$ are displayed in Fig. 4.

Hip Regulators. To account for differences between the physical robot and the ideal model and to stabilize the robot to minor perturbations in the lateral plane, a regulator structure is introduced expanding on previous regulation approaches used on DURUS for flat-footed walking [14]. Discrete logic is used to handle a smooth blending factor $(s)$ increasing linearly through the toe-lift domain $\mathcal{D}_{t l}$ according to the change in the normalized phase variable, $\Lambda$. Throughout all other domains $\left\{\mathcal{D}_{h s}, \mathcal{D}_{t s}, \mathcal{D}_{h l}\right\}$, the blending factor is held constant in order to 


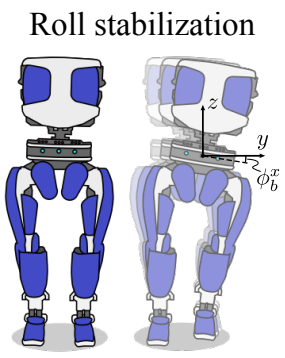

sagittal waist roll $\left(\phi_{b}^{x}\right)$

(a)

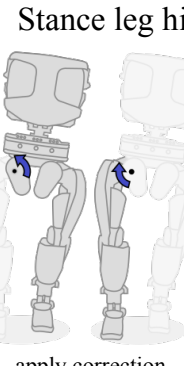

apply correction torque to torso

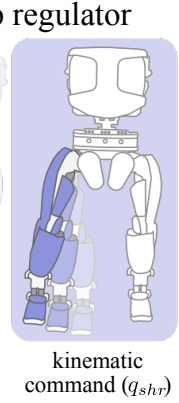

(b)

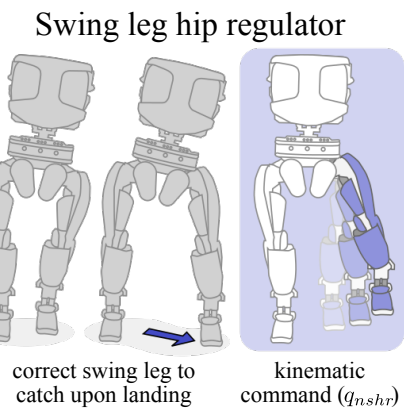

(c)

Fig. 5: Pictured is an illustration of the regulator response to (a) an excessive frontal waist roll. During stance, (b) a counter-rotating torque on the torso is desired to correct the torso roll (left), so a kinematic command is given to the stance leg to adjust the abduction/adduction angle (right). During swing (c) the regulator widens the strike stance between the two legs (left) by kinematically adjusting the swing leg abduction/adduction angle (right).

prevent opposing motions of the legs while both legs are in contact with the ground.

Regulation is provided for two scenarios in which it can compensate for rolling to the outside of the stance leg or towards the swing leg, pictured in Fig. 5. Each regulator performs motion in one direction; adduction for the stance leg and abduction for the swing. Each leg is assigned both a stance and nonstance blending factor for the stance and nonstance hips $s_{i, n s h}$ and $s_{i, s h}$ where $i \in$ \{stance,nonstance $\}$. The stance hip blending factor is increased for the hip currently in stance $\left(s_{s, s h}\right)$ and decreased for the swing hip $\left(s_{n s, s h}\right)$. The converse is true for the swing blending factor. The regulator action for each leg is then:

$$
\begin{aligned}
\Delta q_{i, n s h r}^{d} & =-s_{i, n s h} K_{n s h}\left(y^{a}-y^{d}\right), \\
\Delta q_{i, s h r}^{d} & =-s_{i, s h} K_{s h}\left(y^{a}-y_{d}\right),
\end{aligned}
$$

where $\Delta q_{i, h r}^{d}$ and $\Delta q_{i, n s h r}^{d}$ are the angle abduction and adduction angles added to the trajectories as regulation, $y^{a}:=\phi_{b}^{x, a}$, and $y^{d}:=\phi_{b}^{x, d}$ are the measured waist roll and time based waist roll recorded in simulation, and $K_{n s h, s h}$ are the tunable nonstance hip and stance hip proportional gains.

Ankle Inverse Kinematics. While performing multi-contact walking the heel holonomic constraint is very important, particularly as it impacts the ground and transitions between the domains $\mathcal{D}_{h l}$ and $\mathcal{D}_{h s}$. If the robot strikes the ground with a foot configuration which does not have the heel parallel to the ground, then it will be thrown off balance. To ensure the holonomic constraint at this transition is satisfied, we implement an inverse kinematics solver. Angles for the motors controlling the pushrod transmission in the swing foot are solved with a Newton-Raphson method and ensure the swing foot strikes the ground evenly. 

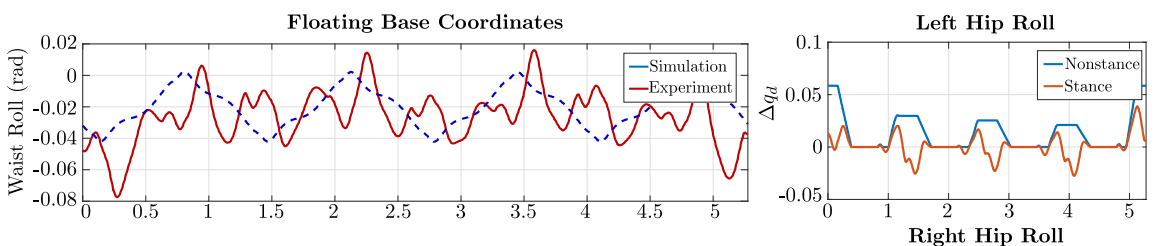

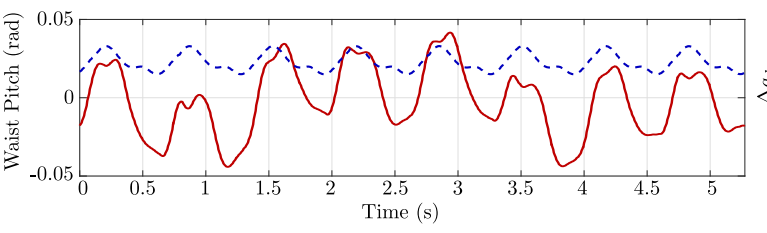

(a)

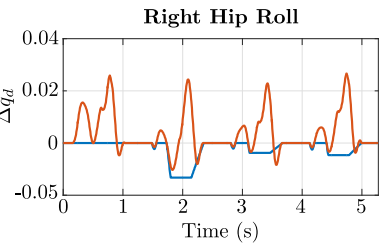

(b)

Fig. 6: A plot of the floating base coordinates (a) measured by IMU (solid) versus the simulated walking data (dashed) and the corresponding stance and swing regulator actions (b) over six steps.

\subsection{Experimental Results}

This section discusses the experimental results which were obtained through the implementation of the walking gait which was found through the multi-domain optimization problem ${ }^{1}$. The limit cycles achieved experimentally on DURUS shown in Fig. 7 exhibit a closed behavior, indicating the multi-contact walking behaviors are stable. It is clear from the hip roll limit cycle that this the joint most heavily augmented by the feedback regulators. These regulators react to the floating base coordinate, pictured in Fig. 6. We can see that while the system has a limit cycle on the floating base coordinates there is a mismatch between the experiment and simulation which is particularly evident in the pitch direction. This mismatch is a key point that the authors hope to address in future work in which more advanced controllers can be applied to the multi-contact gait.

The experimentally implemented walking gait ambulated with a forward velocity of $0.60 \mathrm{~m} / \mathrm{s}$ and a stride length of 0.39 , for which the specific cost of electrical transport (CoT) $c_{e t, i}$ for each step is computed according to [5] as:

$$
c_{e t, i}=\frac{1}{m g d_{i}} \int_{t_{i}^{+}}^{t_{i}^{-}} P_{e l}+\sum_{j=1}^{15} I_{j}(t) V_{j}(t) d t
$$

where $P_{e l}=86.4 \mathrm{~W}$ is logic power consumed by the onboard computer and motor controllers, $d_{i}$ is the x-position traveled by the non-stance foot of the robot through the $i^{\text {th }}$ step, and $I_{j}(t)$ and $V_{j}(t)$ are the currents and voltage recorded for the $j^{\text {th }}$ motor. The mean total power consumed over all 15 actuators for 200 steps, along with the cost of transport per step can be seen in Fig. 8 . These results indicate that the mean CoT for DURUS during steady-state multicontact locmotion is $\bar{c}_{e t}=1.02$, which is $37 \%$ more efficient than experimental results obtained on DURUS for flat-footed walking [14], which was previously the lowest recorded CoT on a humanoid robot.

\footnotetext{
${ }^{1}$ See [1] for a video of DURUS walking with the multi-contact behaviors.
} 


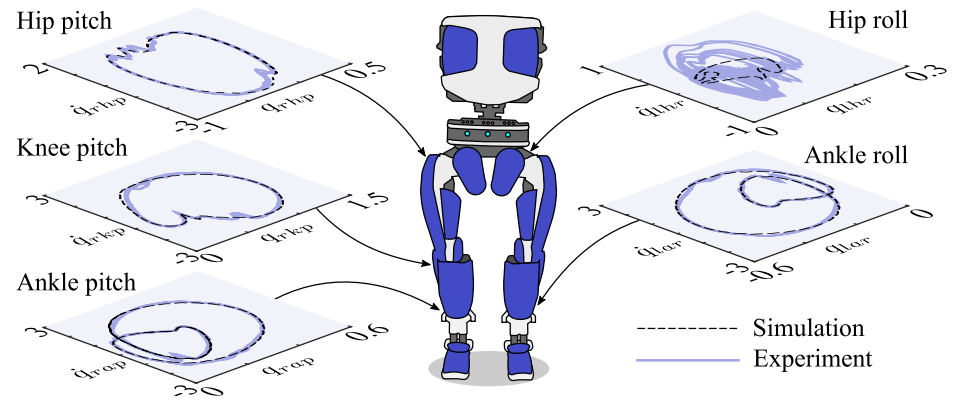

Fig. 7: Pictured is the trace of continuous walking limit cycles over 10 steps (solid) compared to the nominal time based trajectory from simulation (dashed). (Units: $\mathrm{rad}$ and $\mathrm{rad} / \mathrm{s}$ )

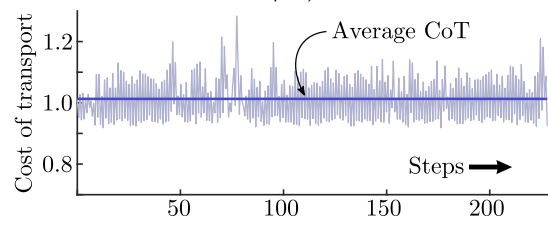

(a)

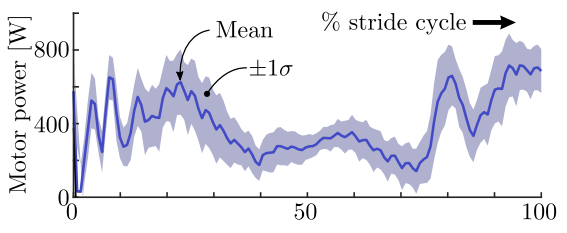

(b)

Fig. 8: (a) The specific cost of transport over 200 steps of continuous walking. (b) Mean motor power consumed per step over 200 step interval.

\section{Methodology Performance}

This section provides some metrics and performance discussion on the overall algorithm. As the primary component in designing these walking gaits, an efficient implementation of the optimization approach proposed in Sec. 3 is crucial to the rapid design of multi-contact walking gaits. The optimization is implemented in MATLAB using the software package IPOPT $^{2}$ with linear solver ma57 on a laptop computer with an Intel Core i7-3820QM processor $(2.7 \mathrm{GHz})$ and 12 GB of RAM. The number of cardinal nodes are picked as 10, 15, 20, and 12 for the toe-strike, toe-lift, heel-strike, and toestrike domains, respectively. Based on the formulation presented in Sec. 3 we arrive at an NLP with 21, 309 variables, 22,721 constraints, and a Jacobian sparsity of $0.05 \%$. Typical computation times for the multi-contact behavior in this work is 647 seconds over 418 iterations.

Since this methodology begins with analysis and utilization of a domain sequencing mirroring that of humans, we would like to better determine whether the approach generates behaviors in line with that of nominal human walking. To provide a more quantitative measure of "human-likeness" of DURUS' walking, the human-based cost of eight human subjects ${ }^{3}$ and DURUS are computed with respect to the nominal human domain cycle presented in Table 1 . We define a walking cycle as a pair $(\gamma, l)$ with $l=(V, E)$ the graph presented in Fig. 3

\footnotetext{
2 https://projects.coin-or.org/Ipopt

3 The human walking cycles analyzed are derived from the dataset presented in [4].
} 


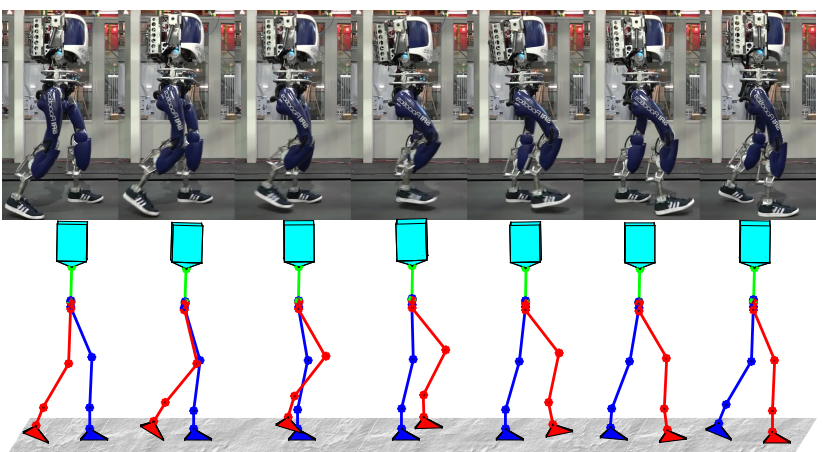

(a)

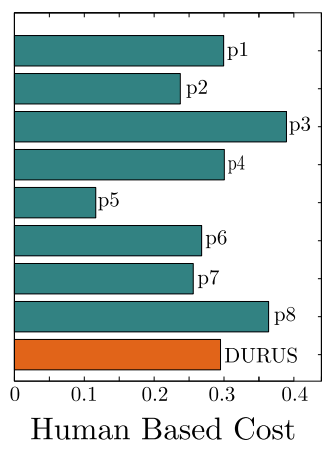

(b)

Fig. 9: (a) Tiles of DURUS walking seen at an angle from front. (b) Human-based cost of DURUS compared to eight healthy human subjects.

and $\gamma: l \rightarrow \mathbb{R}^{|V|}$ is a function such that $\gamma(v) \geq 0$ and $\sum_{v \in V} \gamma(v)=1$. The human-based cost for the multi-contact walking gaits can be found as the cut distance between the optimized cycle and the nominal human cycle. Specifically, let $\left(\gamma^{*}, l^{*}\right)$ be the nominal human walking cycle, and view $\gamma^{*}$ and $\gamma_{r}$ as functions on $V^{*} \cup V_{r}$ by letting $\gamma^{*}(i) \equiv 0$ if $i \in V_{r} \backslash V^{*}$ and $\gamma_{r}(j) \equiv 0$ if $j \in V^{*} \backslash V_{r}$. The cut distance is then computed as in [4] by:

$$
\begin{array}{r}
d\left(\gamma^{*}, l^{*}, \gamma_{r}, l_{r}\right)=\max _{I, J \subset V^{*} \cup V_{r}}\left|\sum_{i \in I, j \in J}\left(\gamma^{*}(i) \gamma^{*}(j) \beta^{*}(i, j)-\gamma_{r}(i) \gamma_{r}(j) \beta(i, j)\right)\right| \\
+\sum_{k \in V^{*} \cup V_{r}}\left|\gamma^{*}(k)-\gamma_{r}(k)\right|,
\end{array}
$$

where $\beta^{*}(i, j)=1$ for all edges $(i, j) \in E^{*}$ and $\beta_{r}(i, j)=1$ for all edges $(i, j) \in$ $E_{r}$. The eight healthy subjects feature human-based costs ranging from 0.12 to 0.36 in which DURUS has a cost of 0.30. A comparison of the human-based costs for each of these subjects and DURUS is pictured in Fig. 9(b).

The end result of the approach presented in this paper is a stable multicontact dynamic walking gait which lends itself to experimental implementation on humanoid robots. Additionally, we have shown that the duration percentages of each discrete domain of the optimal gait match very closely to human walking [4], that is, we have recovered human-like behavior without explicit human reference as a consequence of the natural dynamics of the robot. Walking tiles from the experiment and simulation are synchronized and shown in Fig. 9 (a). While this work primarily focused on the development of periodic multi-contact walking motions on flat terrain it is extensible to the prototyping of other behavior cycles which can be represented within in the framework presented in Sec. 2. This could include alternating step lengths or speeds, stairs, and ramps to name a few. Future work will attempt to apply more rigorous controllers to the walking behaviors generated using this behavior generation methodology. 


\section{Conclusions}

This paper presented a complete algorithmic approach, from modeling to hardware implementation, which was used to obtain multi-contact locomotion on the 3D humanoid robot, DURUS. Through a hybrid model mirroring closely that of a naturally walking human, an optimization problem was formulated for gait generation. Additional constraints allowed for the adjustment of parameters in the gaits generated by the optimizer are introduced and iteratively tuned until a gait is produced which performs well on hardware as a feedforward trajectory playback term. To stabilize the robot when the behaviors are implemented on hardware, three compensation terms are used: a compliance compensator, hip roll proportional regulators, and an inverse kinematics solver which ensures that the swing heel strikes parallel to the ground, thus satisfying the holonomic constraint at the beginning of the following domain. The end result is dynamic and efficient locomotion on the physical hardware utilizing the full-order dynamics of the $3 \mathrm{D}$ humanoid robot. This walking was shown to be both stable and energy efficient, demonstrating an even lower electrical cost of transport than previously attained on DURUS. Therefore, this paper presented an algorithmic framework producing the most efficient walking realized on a humanoid robot that is notably human-like when compared to the breakdown of multi-contact foot behavior present in human locomotion.

\section{Acknowledgment}

The authors would like to SRI for the design and support of DURUS and Eric Ambrose for designing the custom foot used on DURUS in this work. This work is supported by the National Science Foundation through NRI-1526519.

\section{References}

1. DURUS walks like a human. https://youtu.be/1fC7b2LjVW4.

2. M. Ackermann. Dynamics and Energetics of Walking with Prostheses. PhD thesis, University of Stuttgart, Stuttgart, 2007.

3. Aaron D Ames. Human-inspired control of bipedal walking robots. IEEE Transactions on Automatic Control, 59(5):1115-1130, 2014.

4. Aaron D Ames, Ramanarayan Vasudevan, and Ruzena Bajcsy. Human-data based cost of bipedal robotic walking. In Proceedings of the 14 th international conference on Hybrid systems: computation and control, pages 153-162. ACM, 2011.

5. Steve Collins, Andy Ruina, Russ Tedrake, and Martijn Wisse. Efficient bipedal robots based on passive-dynamic walkers. Science, 307(5712):1082-1085, 2005.

6. Jessy W Grizzle, Christine Chevallereau, Ryan W Sinnet, and Aaron D Ames. Models, feedback control, and open problems of 3D bipedal robotic walking. $\mathrm{Au}$ tomatica, 50(8):1955-1988, 2014.

7. Nandha Handharu, Jungwon Yoon, and Gabsoon Kim. Gait pattern generation with knee stretch motion for biped robot using toe and heel joints. In Humanoids 2008-8th IEEE-RAS International Conference on Humanoid Robots, pages 265270. IEEE, 2008. 
8. Ayonga Hereid, Eric A. Cousineau, Christian M. Hubicki, and Aaron D. Ames. 3D dynamic walking with underactuated humanoid robots: A direct collocation framework for optimizing hybrid zero dynamics. In IEEE International Conference on Robotics and Automation (ICRA). IEEE, 2016.

9. Ayonga Hereid, Shishir Kolathaya, Mikhail S Jones, Johnathan Van Why, Jonathan W Hurst, and Aaron D Ames. Dynamic multi-domain bipedal walking with ATRIAS through SLIP based human-inspired control. In Proceedings of the 17th international conference on Hybrid systems: computation and control, pages 263-272. ACM, 2014.

10. Y. Hurmuzlu. Dynamics of bipedal gait; part i: Objective functions and the contact event of a planar five-link biped. ASME Journal of Applied Mechanics, 60(2):331336, 1993.

11. Verne T Inman. Human locomotion. Canadian Medical Association Journal, 94(20):1047, 1966.

12. Matthew Johnson, Brandon Shrewsbury, Sylvain Bertrand, Tingfan Wu, Daniel Duran, Marshall Floyd, Peter Abeles, Douglas Stephen, Nathan Mertins, Alex Lesman, et al. Team IHMC's lessons learned from the DARPA robotics challenge trials. Journal of Field Robotics, 32(2):192-208, 2015.

13. M. Posa, S. Kuindersma, and R. Tedrake. Optimization and stabilization of trajectories for constrained dynamical systems. In 2016 IEEE International Conference on Robotics and Automation (ICRA), pages 1366-1373, May 2016.

14. Jacob Reher, Eric A. Cousineau, Ayonga Hereid, Christian M. Hubicki, and Aaron D. Ames. Realizing dynamic and efficient bipedal locomotion on the humanoid robot DURUS. In IEEE International Conference on Robotics and Automation (ICRA). IEEE, 2016.

15. Ramzi Sellaouti, Olivier Stasse, Shuuji Kajita, Kazuhito Yokoi, and Abderrahmane Kheddar. Faster and smoother walking of humanoid HRP-2 with passive toe joints. In 2006 IEEE/RSJ International Conference on Intelligent Robots and Systems, pages 4909-4914. IEEE, 2006.

16. Ching-Long Shih, JW Grizzle, and Christine Chevallereau. From stable walking to steering of a 3D bipedal robot with passive point feet. Robotica, 30(07):1119-1130, 2012.

17. Koushil Sreenath, Hae-Won Park, Ioannis Poulakakis, and Jessy W. Grizzle. Compliant hybrid zero dynamics controller for achieving stable, efficient and fast bipedal walking on MABEL. International Journal of Robotics Research, 30(9):1170-1193, August 2011.

18. DH Sutherland, KR Kaufman, and JR Moitoza. Kinematics of normal human walking. Human walking, 2:23-44, 1994.

19. Miomir Vukobratović and Branislav Borovac. Zero-moment point thirty five years of its life. International Journal of Humanoid Robotics, 1(01):157-173, 2004.

20. E. Westervelt, J.W. Grizzle, and D.E. Koditschek. Hybrid zero dynamics of planar biped walkers. IEEE Transactions on Automatic Control, 48(1):42-56, January 2003.

21. E. R. Westervelt, J. W. Grizzle, C. Chevallereau, J.-H. Choi, and B. Morris. Feedback Control of Dynamic Bipedal Robot Locomotion. Control and Automation. Boca Raton, FL, June 2007.

22. Huihua Zhao, Ayonga Hereid, Wen-loong Ma, and Aaron D Ames. Multi-contact bipedal robotic locomotion. Robotica, pages 1-35, 2015. 